\title{
Framework for hyperspectral image processing and quantification for cancer detection during animal tumor surgery
}

Guolan Lu

Dongsheng Wang

Xulei Qin

Luma Halig

Susan Muller

Hongzheng Zhang

Amy Chen

Brian W. Pogue

Zhuo Georgia Chen

Baowei Fei

\section{SPIE.}




\title{
Framework for hyperspectral image processing and quantification for cancer detection during animal tumor surgery
}

\author{
Guolan Lu, ${ }^{a}$ Dongsheng Wang, ${ }^{b}$ Xulei Qin, ${ }^{c}$ Luma Halig, ${ }^{c}$ Susan Muller, ${ }^{d}$ Hongzheng Zhang, ${ }^{d}$ Amy Chen, ${ }^{d}$

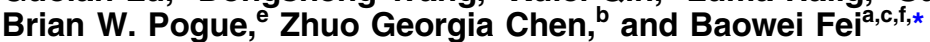 \\ ${ }^{a}$ Georgia Institute of Technology and Emory University, The Wallace H. Coulter Department of Biomedical Engineering, Atlanta, Georgia 30332, \\ United States \\ ${ }^{b}$ Emory University, School of Medicine, Department of Hematology and Medical Oncology, , Atlanta, Georgia 30332, United States \\ 'Emory University, School of Medicine, Department of Radiology and Imaging Sciences, , Atlanta, Georgia 30332, United States \\ 'Emory University, School of Medicine, Department of Otolaryngology, , Atlanta, Georgia 30332, United States \\ ${ }^{e}$ Dartmouth College, Thayer School of Engineering, Hanover, New Hampshire 03755, United States \\ 'Winship Cancer Institute of Emory University, Atlanta, Georgia 30322, United States
}

\begin{abstract}
Hyperspectral imaging $(\mathrm{HSI})$ is an imaging modality that holds strong potential for rapid cancer detection during image-guided surgery. But the data from $\mathrm{HSI}$ often needs to be processed appropriately in order to extract the maximum useful information that differentiates cancer from normal tissue. We proposed a framework for hyperspectral image processing and quantification, which includes a set of steps including image preprocessing, glare removal, feature extraction, and ultimately image classification. The framework has been tested on images from mice with head and neck cancer, using spectra from 450- to 900-nm wavelength. The image analysis computed Fourier coefficients, normalized reflectance, mean, and spectral derivatives for improved accuracy. The experimental results demonstrated the feasibility of the hyperspectral image processing and quantification framework for cancer detection during animal tumor surgery, in a challenging setting where sensitivity can be low due to a modest number of features present, but potential for fast image classification can be high. This HSI approach may have potential application in tumor margin assessment during image-guided surgery, where speed of assessment may be the dominant factor. ( 2015 Society of Photo-Optical Instrumentation Engineers (SPIE) [DOI: 10.1117/1.JBO.20.12.126012]
\end{abstract}

Keywords: hyperspectral imaging; cancer surgery; intra-operative cancer detection; image registration; glare removal; feature extraction; feature selection; mutual information.

Paper 150401RR received Jul. 4, 2015; accepted for publication Nov. 25, 2015; published online Dec. 28, 2015.

\section{Introduction}

It is estimated that there are more than 1.6 million new cancer cases and that there will be 0.5 million cancer deaths in the United States in $2015 .{ }^{1}$ The annual cost for cancer care is projected to rise from $\$ 104$ billion in 2006 to over $\$ 174$ billion in $2020 .^{2}$ Surgery remains the fundamental treatment for most solid tumors. ${ }^{3}$ During surgery, complete tumor removal is essential for the postoperative prognosis of patients. ${ }^{4}$ Positive resection margins could lead to local recurrence of cancer, ${ }^{5}$ additional surgeries, ${ }^{6}$ and increased mortality for cancer patients. ${ }^{7,8}$ Thus, improvement in the completeness of tumor removal would benefit patients and might produce significant cost savings. ${ }^{3}$

During tumor surgery, it can be challenging to differentiate cancer from normal tissue during the process of resection. Visual inspection and palpation are routinely used to determine tumor margins during surgery. ${ }^{9}$ This process can be highly subjective and inconclusive. Intraoperative frozen tissue evaluation is also used to estimate surgical margin at initial surgery, ${ }^{3}$ which may suffer from errors that occur during sampling and histological interpretation, and pressure is always mounting to reduce this time and cost factor. In addition, the histological processing can take time, ${ }^{10}$ which is labor intensive and extends anesthesiarelated risks. Therefore, there are clinical needs to develop real-time imaging methods to help the surgeon for localization and assessment of tumor margins during surgery.

Hyperspectral imaging (HSI) has emerged as a promising modality for medical applications in recent years. ${ }^{11-16}$ It may provide a noninvasive tool for intraoperative tumor visualization. The principle of HSI is to acquire two-dimensional (2-D) spatial images in typically hundreds of contiguous bands of high spectral resolution covering the ultraviolet, visible, and near-infrared (IR) bands. In this way, HSI extends probe-based spectroscopy to spectral imaging, which makes it possible to spatially demarcate the tumor margins. The advantages of HSI include low cost, fast speed, lack of ionizing radiation, and no need of the administration of contrast agents. More importantly, HSI can capture diagnostic information in the ultraviolet and near-IR wavelength regions and extend a surgeon's vision to the invisible region.

Hyperspectral image-guided surgery has been reported in the literature. Panasyuk et al. ${ }^{17}$ utilized HSI to detect small residual tumors of 0.5 to $1 \mathrm{~mm}$ in a breast cancer surgery of a rodent tumor model. Kiyotoki et al. ${ }^{18}$ reported the use of HSI technology for the distinction of gastric cancer from normal mucosa in endoscopically resected lesions. Gebhart et al. ${ }^{19}$ used an imaging system that combined fluorescence and diffuse reflectance imaging for human brain tumor resection guidance. In addition, 
HSI has also been utilized for differentiating key anatomic structures during surgery such as differentiation of the bile duct from surrounding arteries. ${ }^{20}$ A more comprehensive summary about surgical guidance with HSI can be found in our recent review paper. ${ }^{21}$ Although preliminary studies have demonstrated the feasibility of HSI toward an intraoperative visual aid, the diagnostic capability of HSI is largely dependent on the interpretation and development of hyperspectral image analysis methods. The three-dimensional (3-D) dataset acquired by HSI is called a hypercube. Each pixel in a hypercube has a spectral signature that characterizes the composition of that pixel and correlates with the biochemical and morphological changes in tissue. The spectral signature of each pixel in the images provides a clue to noninvasively distinguish cancer from normal tissue. Previous efforts have been mainly focused on the design and testing of various HSI systems, while the preprocessing and analysis of surgical hyperspectral images are not well investigated. The challenges in developing a diagnostic support system for rapid cancer detection mainly lie in two aspects: first, the intraoperative hyperspectral images are usually distorted due to motion artifacts, glare (also known as specular reflection), and curvature variations, which require rigorous preprocessing procedures before further analysis. Second, the analysis of the hyperspectral dataset is time consuming due to the large volume and high dimensionality. On the other hand, real-time surgical guidance requires the analysis to be complete within a certain time frame. Thus, it is essential to extract and select the most relevant features to reduce dimensionality without sacrificing diagnostic accuracy.

The objectives of this study were to (1) develop a set of preprocessing techniques to reduce intraoperative image distortions, (2) conduct feature extraction and selection to identify the most relevant spectral features and reduce data dimensionality, and (3) evaluate the diagnostic potential of the selected feature set for cancer detection. The major contribution is the development of a comprehensive framework for surgical hyperspectral image processing and analysis, which could facilitate the advancement of medical HSI toward clinical translation.

\section{Materials and Experimental Design}

\subsection{Hyperspectral Imaging Instrumentation}

An in vivo small animal imaging system called Maestro (PerkinElmer Inc., Waltham, Massachusetts) was used for acquiring the hyperspectral dataset. This system mainly consists of the light source, wavelength dispersion device, and area detector. A Cermax-type, 300-W xenon light source is used for white-light excitation, which spans the electromagnetic spectrum from 450 to $800 \mathrm{~nm}$. The interior near-IR light source can be used to extend the spectral range of excitation up to $950 \mathrm{~nm}$. Four fiber optic, adjustable illuminator arms yield an even light distribution to the subject. The light from the excitation source illuminates the subject. The reflected light from the surface is split into a series of narrow spectral bands by liquid crystal tunable filters with a bandwidth of $20 \mathrm{~nm}$ and collected by a 12-bit, high-resolution charge-coupled device. The hyperspectral camera can simultaneously acquire a full dataset from as many as three mice, with a spatial resolution of $25 \mu \mathrm{m} /$ pixel, in only a few seconds. The acquisition wavelength region ranges from 450 to $950 \mathrm{~nm}$, with varying step sizes such as 2 , 5, or $10 \mathrm{~nm}$.

\subsection{Animal Imaging and Tumor Surgical Experiments}

A head and neck tumor xenograft model using head and neck squamous cell carcinoma (HNSCC) cell line M4E with green fluorescence protein (GFP) ${ }^{22}$ was adopted in the experiment. The HNSCC M4E cells were maintained as a monolayer culture in Dulbecco's modified Eagle's medium/F12 medium (1:1) supplemented with $10 \%$ fetal bovine serum. ${ }^{14}$ M4E-GFP cells, which are generated by transfection of the pLVTHM vector into M4E cells, were maintained in the same condition as M4E cells. The animal experiment was approved by the Animal Care and Use Committee (IACUC) of Emory University.

In this experiment, eight female nude mice aged 4 to 6 weeks were injected with $2 \times 10^{6}$ M4E cells with GFP on the lower back. Surgery was performed $\sim 1$ month after tumor cell injection. Before surgery, mice were anesthetized with a continuous supply of $2 \%$ isoflurane in oxygen. After the anesthesia administration, the skin covering the tumor was removed to expose the tumor to simulate a surgical situation.

\subsubsection{Reflectance image acquisition}

Hyperspectral images with the interior IR, the white excitation, and an autosetting for exposure time were captured over the exposed tumor. Each hypercube contain 226 spectral bands from 450 to $900 \mathrm{~nm}$ with 2-nm increment.

\subsubsection{Fluorescence image acquisition}

Subsequently, a 450-nm excitation filter and autoexposure time were selected for the blue fluorescence image acquisition. Tumors manifested themselves as green light in the fluorescence images due to GFP.

\subsubsection{Surgical removal of tumors and histology processing}

After imaging, the tumors were removed horizontally from the bottom using a blade and were inked with four different colors to represent the head, tail, left, and right orientations of the tumors in the mice. Histological samples were kept in formalin and sent for histological evaluation after $24 \mathrm{~h}$. Histological slides with hematoxylin and eosin $(\mathrm{H} \& \mathrm{E})$ staining were digitized to provide histological assessment of tumor margins.

\subsection{Evaluation Methods for Cancer Detection}

In this study, GFP fluorescence images provide the in vivo gold standard for tumor margin delineation. Although the current gold standard for cancer diagnosis remains histological assessment of H\&E stained tissue, the ex vivo tissue specimen undergoes deformations, including shrinkage, tearing, and distortion, which makes it difficult to align the ex vivo gold standard with in vivo tumor tissue. However, the in vivo GFP fluorescence images provided a much better alignment with hyperspectral reflectance images in the animal model, since they were acquired in vivo immediately after the acquisition of reflectance images for each mouse while the tumor position and shape in the reflectance images are exactly the same as those in the fluorescence images. The tumor and surrounding normal tissue exhibited high contrast in the GFP images. Since histopathology is the gold standard for cancer diagnosis in clinics, we also acquired the H\&E stained histological image as the ex vivo gold standard. 
In this paper, tumor regions were identified manually on the GFP images, and the classification results were then compared with the manual maps. Since human tissue does not contain GFP naturally, registration methods are desirable to align the in vivo hyperspectral images with ex vivo histological images as discussed in our previous publication ${ }^{23}$ in order to move forward for future human studies.

\section{Framework for Hyperspectral Image Processing and Quantification}

Although hyperspectral image analysis methods have been developed for over three decades in the remote sensing area, they are still in their early stage in biomedical applications. With high dimensional datasets, it is not a trivial task to extract the most relevant information from the raw data and classify it into tumor and nontumor tissue. Automated intraoperative cancer detection is especially challenging due to the motion artifacts and glares caused by specular reflection ${ }^{24}$ and the high dimensionality of the dataset. ${ }^{15}$ Therefore, we proposed a comprehensive workflow, as shown in Fig 1, which includes a set of preprocessing techniques as well as feature extraction and selection methods for intraoperative hyperspectral image analysis. The performance of the selected feature set is evaluated by a supervised classification method.

\subsection{Preprocessing Methods for Hyperspectral Images}

The preprocessing steps of the hypercube consist of data normalization, image registration, glare detection and removal, and curvature correction.

\subsubsection{Reflectance image calibration}

The purpose of data normalization was to remove the spectral nonuniformity of the illumination device and the influence of the dark current. The white reference cube was acquired by placing a standard white reference in the field of view, with the white excitation source, interior IR source, and autoexposure time setting. The dark reference cube was captured by keeping the camera shutter closed. The raw data can be corrected by converting into relative reflectance data in Eq. (1) as follows:

$I_{\text {reflectance }}(\lambda)=\frac{I_{\text {raw }}(\lambda)-I_{\text {dark }}(\lambda)}{I_{\text {white }(\lambda)}-I_{\text {dark }}(\lambda)}$.

\subsubsection{Registration of hyperspectral images for motion correction}

The HSI instrument used in our study was a spectral-scanning system, which captured the whole scene with 2-D detector arrays in a single exposure and then stepped through wavelengths to complete the 3-D data cube. The exposure time for each image band varies between 12 and $30 \mathrm{~ms}$, so the acquisition of one hypercube takes about 4 to $7 \mathrm{~s}$. The breathing and heart motion of the mice during the image acquisition would lead to misalignment of the images at different bands even though they are within the same hypercube, which would affect the shape and intensity of the spectral curve. In consequence, the distinction between cancer and normal tissue with pixelwise classification approaches may be compromised. Therefore, image registration is required to align the image bands within each hypercube.

Intensity-based rigid registration was employed since the motion was mostly global movement such as translation. The sum of the squared difference was chosen as the similarity metric since it reflected the movement of each pixel very well. Next, a reference image was selected and geometric transformation were applied to the other images so that they are aligned with the reference. Three methods were compared to choose the most suitable reference image: (1) register each image band to its neighboring image band, (2) register all image bands to the band with highest signal-to-noise ratio, and (3) register all image bands to the average of all image bands. The third method was able to remove most of the movement within one hypercube and outperformed the first two methods.

After the reflectance hypercube was registered, the corresponding fluorescence image at the GFP emission band also needed to be aligned to the reflectance hypercube. We registered the GFP image at the peak emission band to the mean of the reflectance hypercube and then manually outlined the tumor border in the registered GFP image. These tumor maps served as the gold standard for the supervised classification in this study.

\subsubsection{Glare removal method for hyperspectral images}

Glare, also called specular reflection, is the mirror-like reflection of incident light from a moist surface. Optical images such as those from an endoscope and colposcopy images acquired during surgery are often strongly affected by glare spots in the images, which presents a major problem for surgical image analysis. ${ }^{24}$ In HSI, glare alters the intensities of the pixels in each image band and consequently changes the spectral

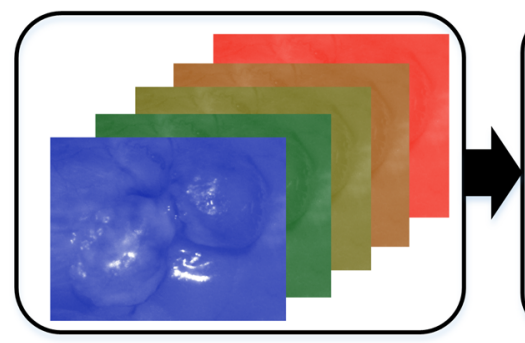

Input hypercube

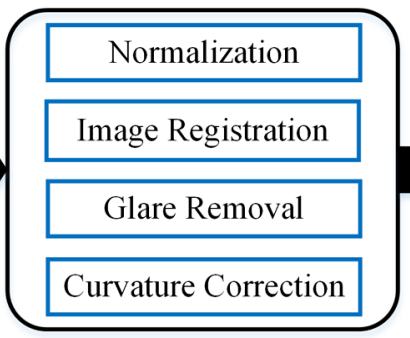

Pre-processing

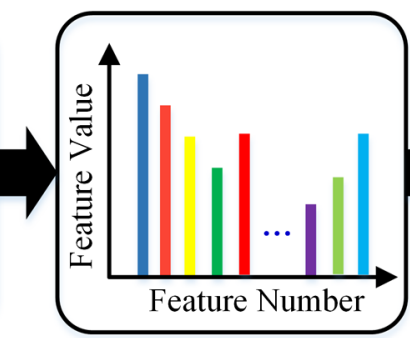

Feature Extraction

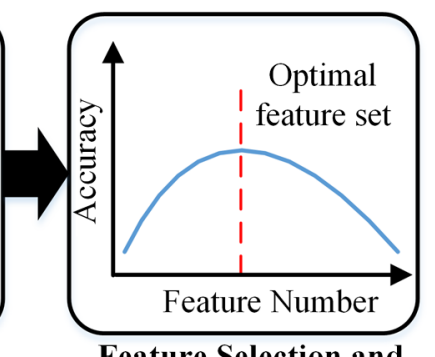

Feature Selection and Classification

Fig. 1 Flowchart of the proposed method. 
fingerprint, which could introduce artifacts in feature extraction and hence deteriorate classification results. Therefore, glare pixels need to be detected and removed before feature extraction and classification.

Currently, no glare detection technique exists for intraoperative hyperspectral images. To better understand the difference of glare pixels, we compared the spectral fingerprint of glare pixels with nonglare pixels and identified the characteristics of glare pixels. It can be seen from Fig. 2 that glare pixels not only had higher intensities but also showed larger variations along the spectral bands. Therefore, the first-order derivatives of the spectral curves of glare pixels were much larger than those of nonglare pixels, as shown in Fig. 2(d).

Based on the above observations, we proposed a three-step glare detection method: (1) estimate the first-order derivatives of spectral curves with a forward difference method. (2) Calculate the standard deviation (std) of each derivative curve and generate an std image for each hypercube. Glare pixels show higher std than normal pixels. (3) Compute the intensity histogram of each std image, fit the histogram with a "log-logistic" distribution (MATLAB function), and experimentally identify the threshold that separates glare and nonglare pixels.

\subsubsection{Curvature correction for hyperspectral images}

In clinical applications, curvature correction is particularly useful when the surface areas of the cancer are raised or depressed with respect to the surrounding tissue. For example, the normal colon tissue surface has numerous folds, and normalization has to be applied to compensate for the difference in the intensity of the light recorded by the camera as a function of tissue geometry. ${ }^{25}$ In our experiment, the exposed tumor surface was raised compared to its surrounding normal tissue. Thus, it was desirable to perform curvature correction to compensate for the spectral variations caused by the elevation of the tumors. The light intensity changes could be viewed as a function of the distance and the angle between the surface and the detector. Two spectra of the same point acquired at two different distances and/or inclinations will have the same shape but vary by a constant. ${ }^{25}$ Dividing each individual spectrum by a constant calculated as the total reflectance across the wavelength range removes the distance and angle dependence as well as dependence on an overall magnitude of the spectrum. This normalization step ensures that the variation in spectra curves is only a function of wavelength, and therefore the differences between cancerous

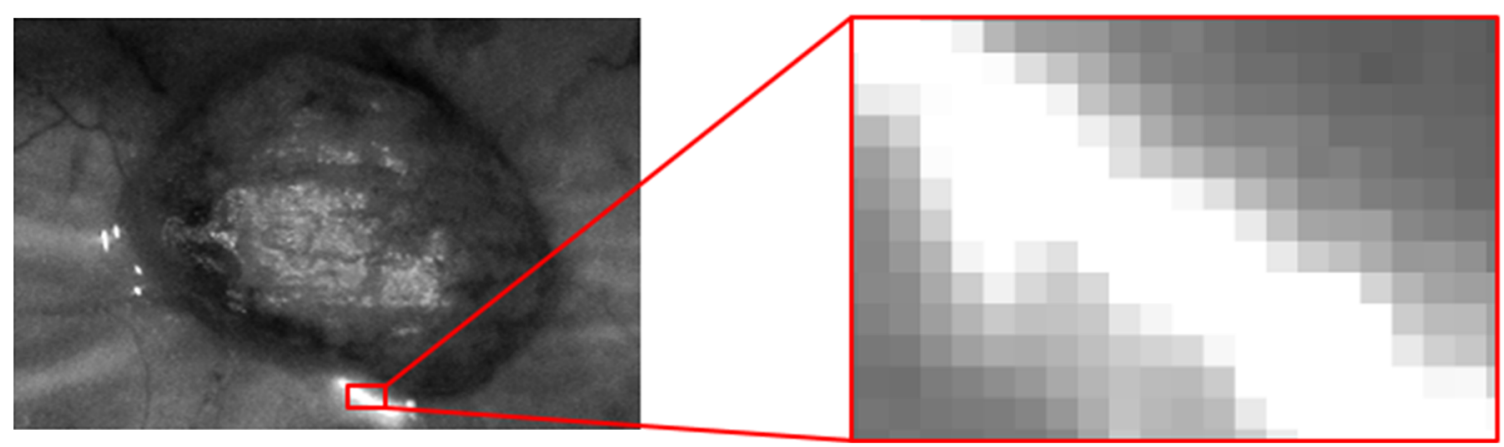

(a)

(b)

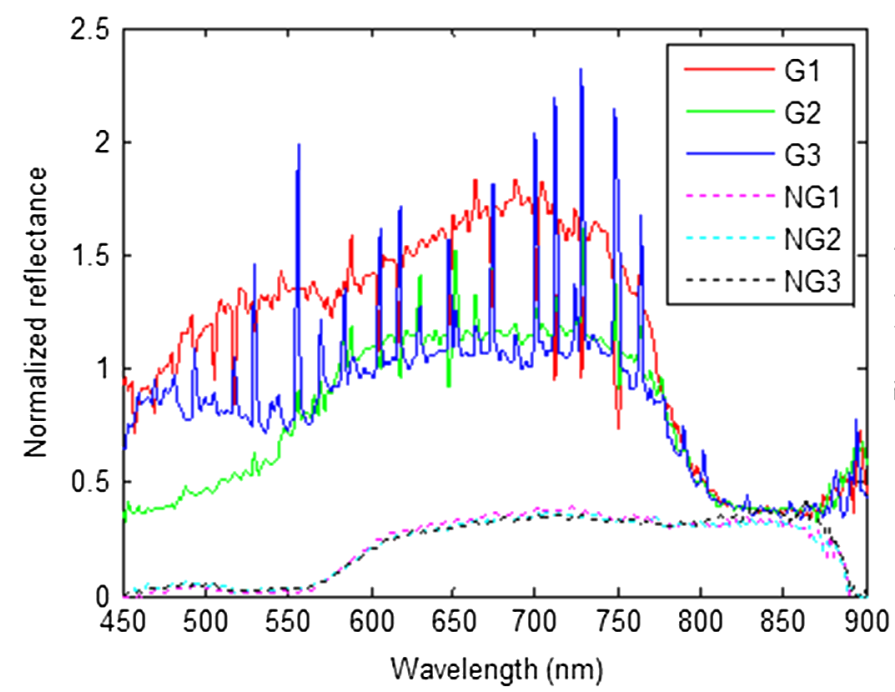

(c)

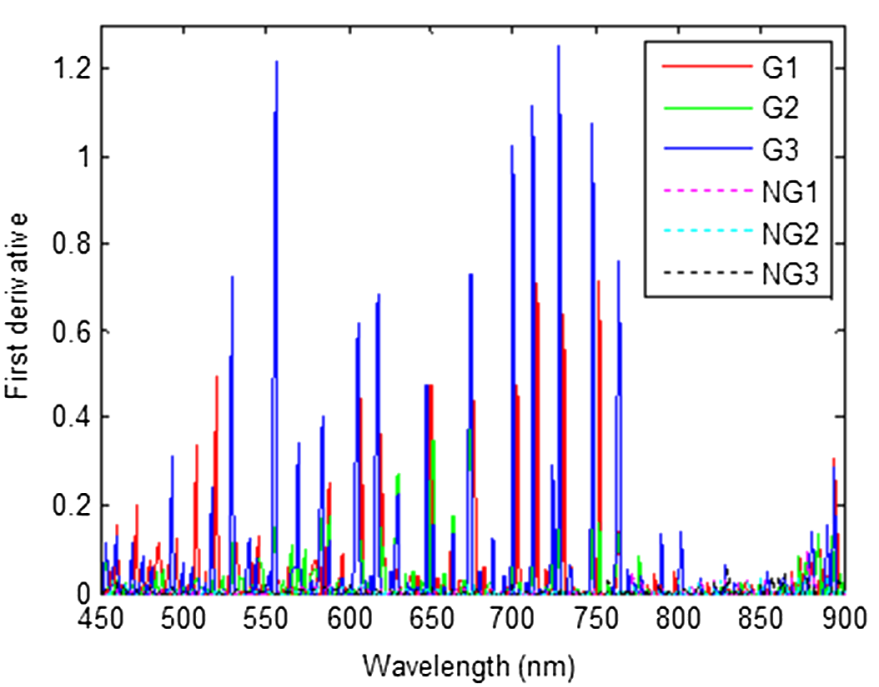

(d)

Fig. 2 Rationale for the proposed glare detection method. (a) Image band at $758 \mathrm{~nm}$. (b) Enlarged image of a selected glare region in the image band in (a). Glare pixels are much brighter than nonglare pixels. (c) Normalized reflectance curve of glare pixels (G1 to G3) and nonglare pixels (NG1 to NG3). Spectral curve of glare pixels varies significantly in many wavelengths. (d) First-order derivative curves corresponding to the spectral curves shown in (c). 
and normal tissue are not affected by the changing curvature of tumors.

\subsection{Feature Extraction from Hyperspectral Data}

Feature extraction and representation is a crucial step for image classification tasks. Efficient feature extraction could lead to improved classification performance. However, HSI has only recently been applied to medical applications, and it is not well understood what features are the most effective and efficient to differentiate cancer from normal tissue in medical hyperspectral images. Currently, one of the most frequently used features is the normalized reflectance curve of each pixel. With only the reflectance features, classification performance is still far from optimal. Therefore, there is still space for adding new features to improve the distinction of cancer from normal tissue.

In this study, we explored the utility of several spectral features, which are derived from the reflectance curve of each pixel. Spatial features are not explored in this study but will be included in our future work. The extracted spectral features included (1) first-order derivatives of each spectral curve, which reflect the variations of spectral information across the wavelength range; (2) second-order derivatives of each spectral curve, which reflect the concavity of the spectral curve; (3) mean, std, and total reflectance at each pixel, which summarize the statistical characteristics of the spectral fingerprint; and (4) Fourier coefficients (FCs), which were initially found to be effective for target detection in the remote sensing field ${ }^{26}$ and later were adopted for breast cancer margin classification from $e x$-vivo breast cancer hyperspectral images. ${ }^{27}$

FC feature extraction involves transforming the original spectral $f(n), n=0,2, \ldots N-1$ into the Fourier domain as $F(k)=\sum_{n=0}^{N-1} s(n) e^{-\frac{2 \pi i}{N} k n}$ and then combining the selected real and imaginary components of FCs $F(k)$ using the following formula:

$\mathrm{FC}(\mathrm{x})=R(x)+I(x)$,

where

$\mathrm{R}(\mathrm{x})=\left\{\begin{array}{ll}\operatorname{Re}\{F(x)\}, & \text { if } \operatorname{Re}\{F(x)\} \geq \operatorname{Im}\{F(x)\} \\ 0, & \text { Otherwise }\end{array}\right.$,

and

$\mathrm{I}(\mathrm{x})= \begin{cases}\operatorname{Im}\{F(x)\}, & \text { if } \operatorname{Im}\{F(x)\} \geq \operatorname{Re}\{F(x)\} \\ 0 & \text { Otherwise }\end{cases}$

Different features may have very different numerical ranges, so each feature was standardized into its z-score (MATLAB function) by subtracting the mean from each feature and then dividing by its std.

\subsection{Feature Selection Method}

After feature extraction, the spectral feature dimension was increased from 226 to 904 . Such a high spectral dimension poses significant challenges to the analysis of the hypercube. High dimensionality can significantly increase the computational burden and storage space, leading to increased data processing time, which is against the requirement of real-time tumor detection during surgery. Depending upon the wavelength range of imaging systems, different studies may have different reflectance features. It is not clear which wavelength is more relevant in characterizing cancerous tissue and would provide a better contrast between cancer and normal tissue. In addition, with a narrow wavelength increment, there is likely spectral redundancy between adjacent bands. Last but not least, increasing the feature dimensionality without increasing the number of training samples may lead to a decrease in classification performance due to the curse of dimensionality, that is, the Hughes phenomenon..$^{28}$ Therefore, it is desirable to analyze the spectral redundancy in the high dimensional data and select the most characterizing compact feature set.

The goal of the feature selection is to find a feature set $S$ with $n$ wavelengths $\left\{\lambda_{i}\right\}$, which optimally characterize the difference between cancerous and normal tissue. To achieve the optimal condition, we used the maximal relevance and minimal redundancy (mRMR) ${ }^{29}$ framework to maximize the dependency of each spectral feature on the target class labels (tumor or normal) and minimize the redundancy among individual features simultaneously. Relevance is characterized by mutual information $\mathrm{I}(x ; y)$, which measures the level of similarity between two random variables $x$ and $y$ :

$\mathrm{I}(x ; y)=\iint p(x, y) \log \frac{p(x, y)}{p(x) p(y)} \mathrm{d} x \mathrm{~d} y$,

where $p(x, y)$ is the joint probability distribution function of $x$ and $y$, and $p(x)$ and $p(y)$ are the marginal probability distribution functions of $x$ and $y$, respectively.

We represent each pixel with $M$ features $\Lambda=$ $\left\{\lambda_{\mathrm{i}}, i=1, \ldots M\right\}, M=904$, and the class label (tumor or normal) with $c$. Then the $\mathrm{mR}$ condition is to search features satisfying Eq. (3) which maximize the mean value of all mutual information values between individual features $\lambda_{i}$ and class $c$ :

$\max D(s, c), \mathrm{D}=\frac{1}{|S|} \sum_{\lambda_{i} \in S} I\left(\lambda_{i}, c\right)$.

The features selected by the $\mathrm{mR}$ condition are likely to have redundancy, which means that the dependency among these features could be large. When two features highly depend on each other, the respective class-discriminative power would not change much if one of them were removed. So, the minimal redundancy condition can be added to select mutually exclusive features:

$$
\min R(s), R=\frac{1}{|S|^{2}} \sum_{\lambda_{i}, \lambda_{j} \in S} I\left(\lambda_{i}, \lambda_{j}\right) .
$$

The simple combination [Eqs. (5) and (6)] of these two conditions forms the criterion mRMR, which can optimize $D$ and $R$ simultaneously:

$$
\max \Phi(D, R), \Phi=D-R
$$

In practice, incremental search methods can be used to find the near-optimal features defined by $\Phi(\cdot)$. Suppose we already identified a feature set $S_{m-1}$ with $m-1$ features. The task is to select the $m$ th feature from the set $\left\{\Lambda-S_{m-1}\right\}$. This is done by selecting the feature that maximizes $\Phi(\cdot)$. The respective incremental algorithm optimizes the following condition: 


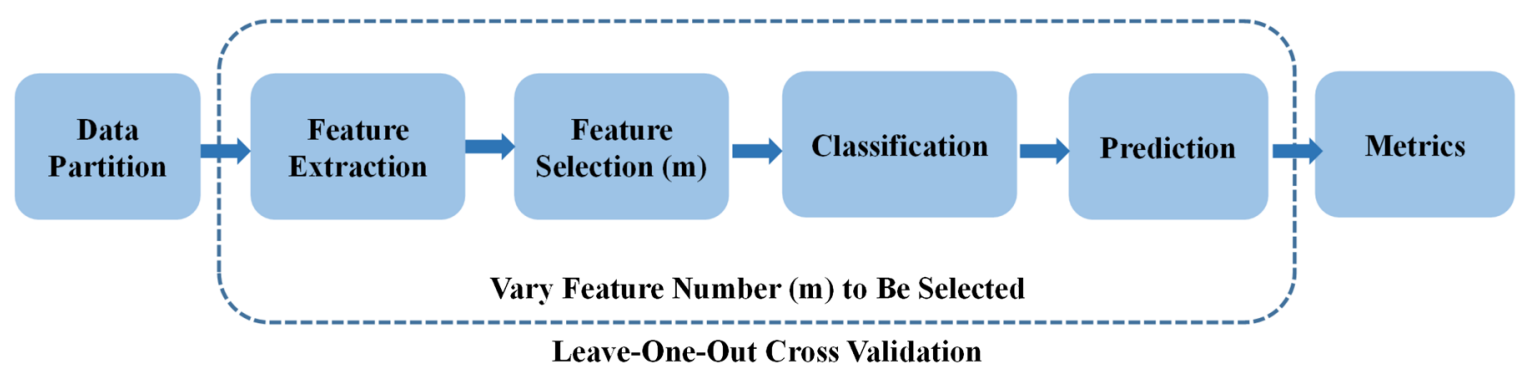

Fig. 3 Flowchart for feature selection and classification.

$$
\max _{\lambda_{j} \in \Lambda-S_{m-1}}\left[I\left(\lambda_{j} ; c\right)-\frac{1}{m-1} \sum_{\lambda_{i} \in S_{m-1}} I\left(\lambda_{j} ; \lambda_{i}\right)\right] .
$$

\subsection{Hyperspectral Image Classification}

To determine the optimal feature set, a K-nearest neighbor (KNN) classifier was employed to evaluate the effectiveness of the selected features with leave-one-out cross validation. The workflow of the feature selection and classification was shown in Fig. 3. First, eight hypercubes were partitioned into a training dataset with seven hypercubes and a testing dataset with the rest of the hypercube. Next, 904 features were extracted from the training data, and mRMR was used to select the optimal feature set on the training dataset as the feature number varied from 1 to 904 . The selected feature set was applied to the testing dataset correspondingly. Next, the KNN classifier was used to train the training data and to predict the labels of the testing dataset with feature sets of different sizes. Finally, the feature set that gave the best classification performance was chosen as the optimal feature set for differentiating cancer from normal tissue.

Accuracy, sensitivity, specificity, and F-score, as defined in Refs. 30 and 31, were chosen as the metrics to evaluate the classification performance of the features. Accuracy represents the percentage of the correctly detected tumor and normal pixels relative to the total number of tumor and normal pixels in an image, respectively. Sensitivity represents the percentage of correctly detected tumor pixels relative to the total number of tumor pixels in an image. Specificity is the percentage of correctly identified nontumor pixels relative to the total number of nontumor pixels in an image. F-score is the harmonic mean of precision and sensitivity.

\section{Experimental Results}

\subsection{Results on Glare Detection and Removal}

Figure 4 shows one example of glare detection results. The bright area in the std image represents the location of glare pixels, which were characterized by large spectral variations and distributed mostly along the long tail of the histogram. The key issue was to identify an appropriate threshold on the histogram that could separate the glare pixels from nonglare pixels in the std images. We compared the traditional thresholding method such as the Ostu method ${ }^{32}$ and entropy method ${ }^{33}$ with the proposed loglogistic curve fitting method. The Ostu and entropy methods were most suitable for histograms with a bimodal shape, but the histogram here contained a very long tail, which was not in the typical bimodal shape. The curve fitting methods produced much a lower threshold than the Ostu and entropy methods, which enabled the detection of the relatively bright glare margins as well as the isolated glare pixels. The threshold was set to be the intensity value, which yielded a certain percentage $(\varepsilon)$ of the peak value in the fitted loglogistic distribution curve. The value of $\varepsilon$ was experimentally set to 5\% through trial and error, which was found sufficient to detect most of the glare pixels. After the glare masks were generated, glare pixels along all the spectral bands were removed from the training and testing datasets, since they did not contain useful diagnostic information.

\subsection{Results on the Comparison Between Green Fluorescent Protein and Non-Green Fluorescent Protein Images}

Figures 5 and 6 show the exposed tumors under the white excitation with and without GFP, respectively. GFP emission peaks under blue excitation occur at the wavelengths of 508 and $510 \mathrm{~nm}$. It was found that under white excitation, the spectral images at these two bands did not exhibit enhanced contrast between tumor and normal tissue compared to other spectral bands. This is consistent with the observation in our previous study. ${ }^{30}$ Therefore, we did not remove GFP spectral bands at 508 and $510 \mathrm{~nm}$ in the preprocessing steps.

Both figures demonstrated that hyperspectral imaging can probe vessels at different depths below the visual surface of the tumor. Light at a shorter wavelength region of the visible range is more sensitive to superficial vascular information due to limited light penetration into the tissue. As the wavelength becomes longer, information from deeper tissue can be acquired. Hence, changing the illumination wavelength may enhance vascular contrast and allow visualization of angiogenesis at the tumor region.

The RGB color image shows the highly vascularized tumors. Figures 5 and 6(c) illustrate the characteristic spectrum of hemoglobin at 542 and $577 \mathrm{~nm}$. These characteristics may contribute to the distinction between cancerous and normal tissue by HSI.

\subsection{Results on Feature Extraction and Visualization}

The most commonly utilized feature for cancer detection with HSI is the normalized reflectance spectra, which reflects the physiological and pathophysiological state of tissue at each pixel. However, this feature alone may not have enough discriminative information to minimize the classification error. It would be very interesting to explore the usefulness of other features besides reflectance. We derived a series of features based on the spectral curve of each pixel and boosted the original feature dimension from 226 to 904. 


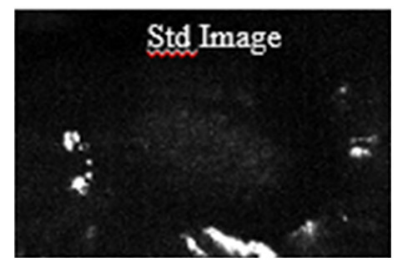

(a)

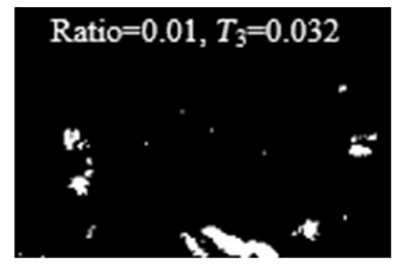

(d)

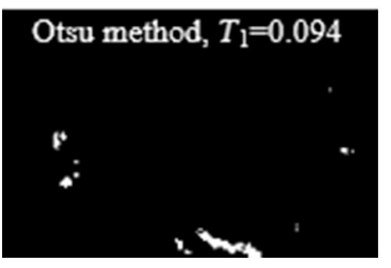

(b)

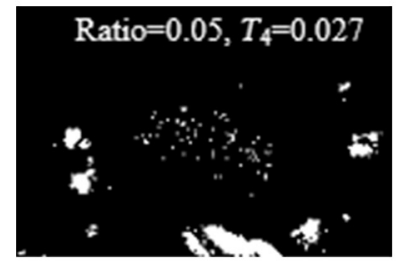

(e)

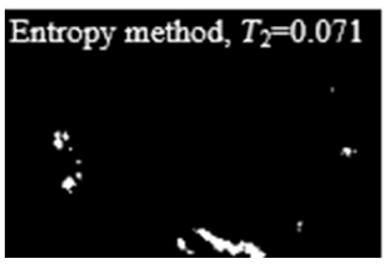

(c)

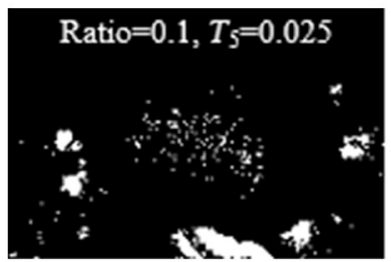

(f)

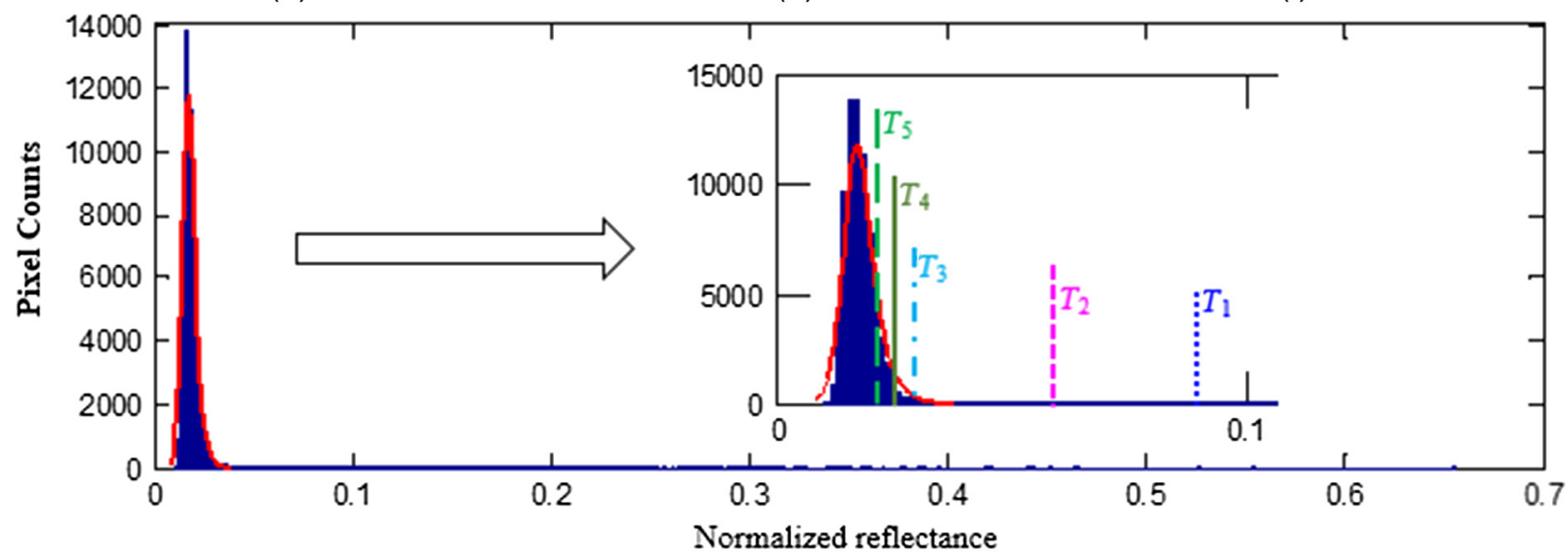

(g)

Fig. 4 Glare detection results: (a) Standard deviation (std) image of the first order derivative for a hypercube. (b) Binary glare map generated by the classical Otsu method. (c) Binary glare map generated by the entropy method. (d)-(f) Glare map generated by the proposed method with ratios $0.01,0.05$, and 0.1 . (g) Histogram of the std image with blue color and loglogistic fitting curve with red color. The five vertical lines represent the five thresholds generated by the five methods in $(b)-(f)$.

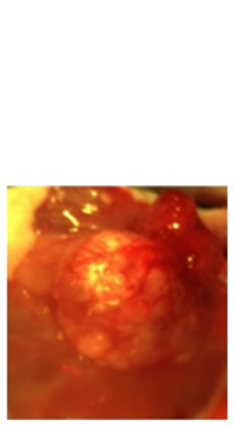

(a)

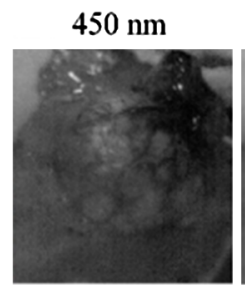

$554 \mathrm{~nm}$

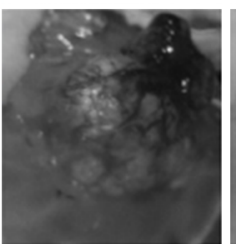

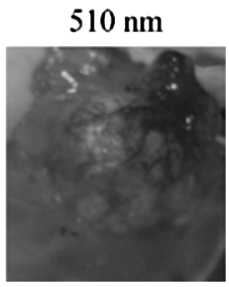

$600 \mathrm{~nm}$

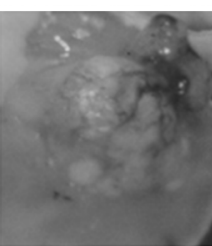

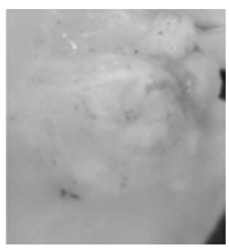

(b)
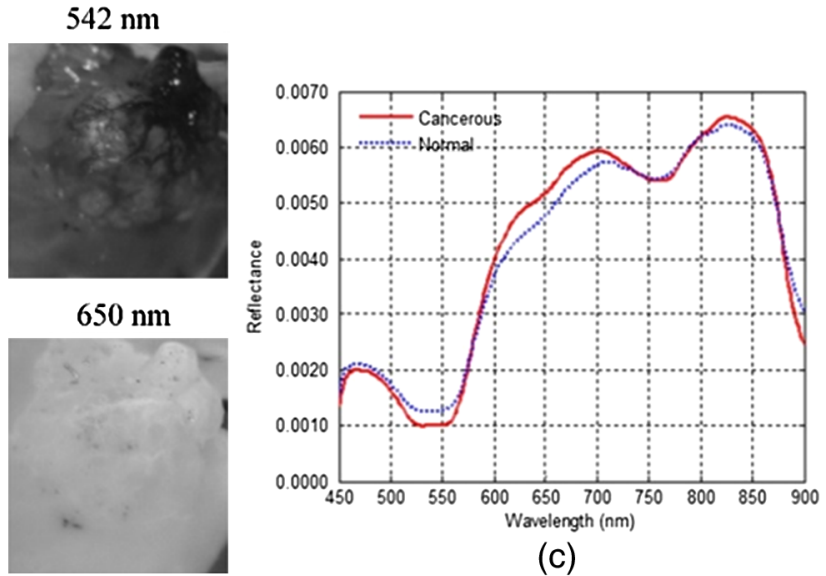

(c)

Fig. 5 Visualization of a tumor with green fluorescence protein (GFP). The upper part is the image of the tumor in a mirror. (a) RGB composite image of the hypercube. (b) Preprocessed spectral images at wavelengths $450 \mathrm{~nm}, 508 \mathrm{~nm}, 510 \mathrm{~nm}, 542 \mathrm{~nm}, 554 \mathrm{~nm}, 576 \mathrm{~nm}, 600 \mathrm{~nm}$, and $650 \mathrm{~nm}$. (c) Spectral curve of cancerous and healthy tissue. 


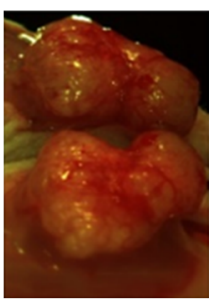

(a)

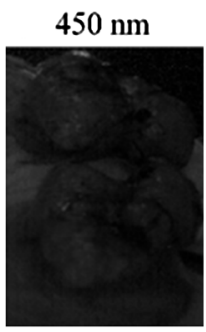

$554 \mathrm{~nm}$

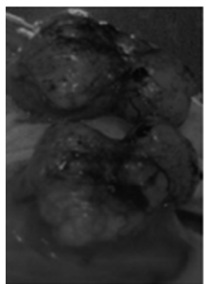

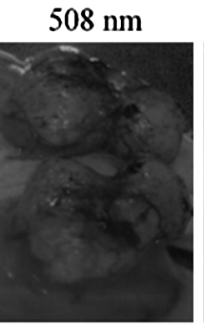

$576 \mathrm{~nm}$

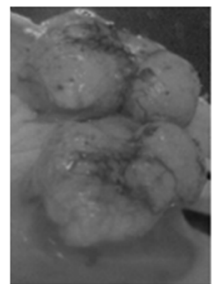

$510 \mathrm{~nm}$

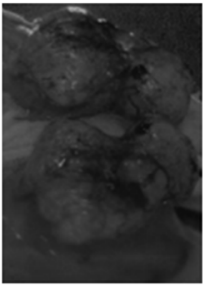

$600 \mathrm{~nm}$

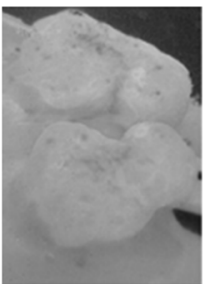

(b)

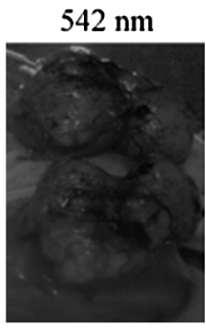

$650 \mathrm{~nm}$

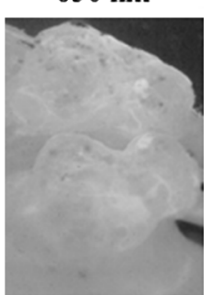

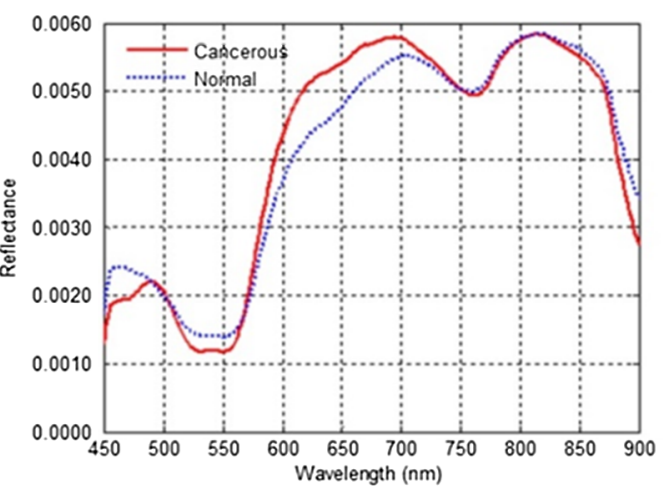

(c)

Fig. 6 Visualization of a tumor without GFP. (a) RGB composite image of the hypercube. (b) Preprocessed spectral images at wavelengths $450 \mathrm{~nm}, 508 \mathrm{~nm}, 510 \mathrm{~nm}, 542 \mathrm{~nm}, 554 \mathrm{~nm}$, $576 \mathrm{~nm}, 600 \mathrm{~nm}$, and $650 \mathrm{~nm}$. (c) Spectral curve of cancerous and healthy tissue.
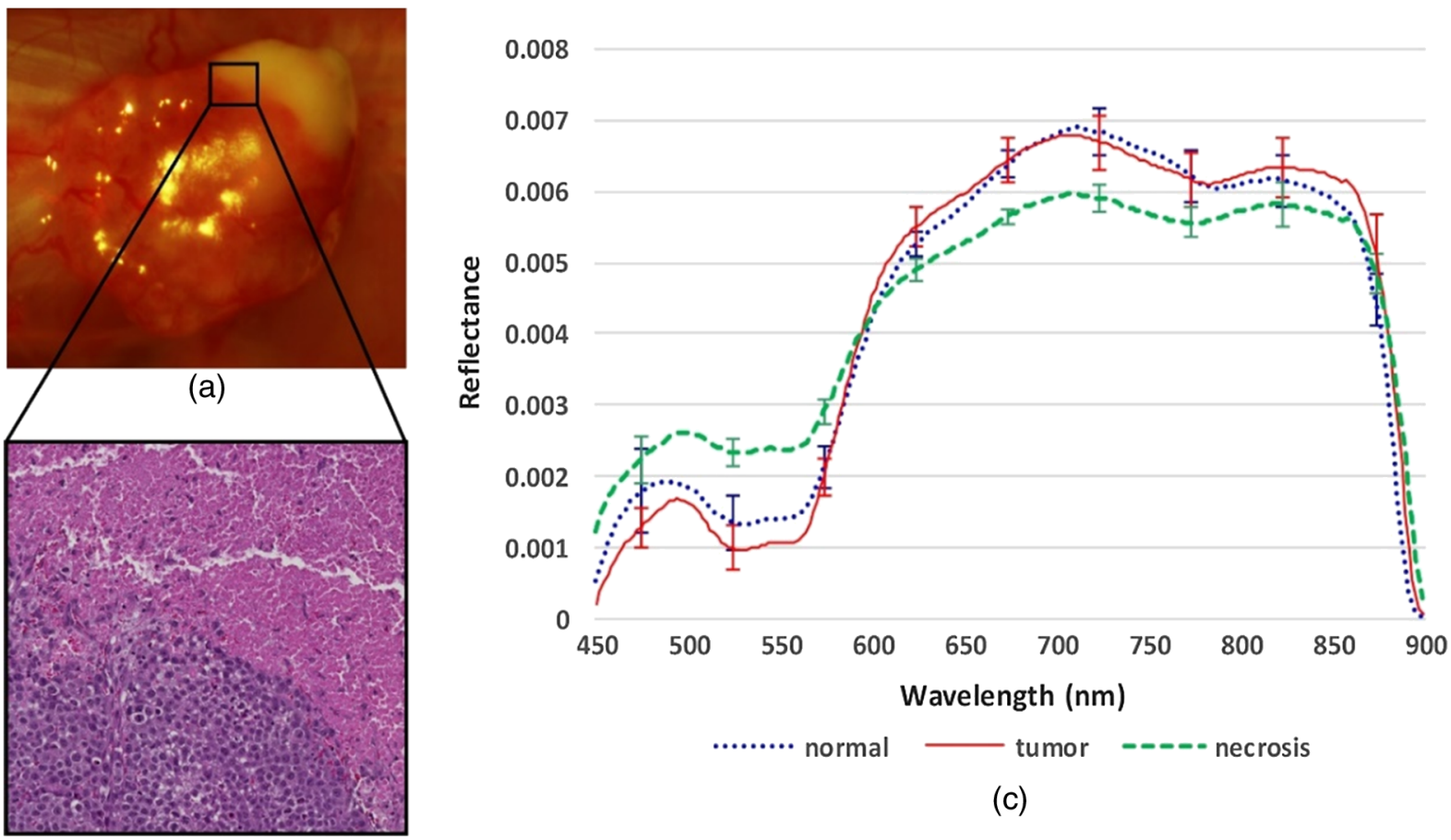

(c)

(b)

Fig. 7 Reflectance spectral curve of a tumor with necrosis. (a) RGB composite image of hypercube. The white region looks necrotic, and the other part of the tumor contains many vessels; (b) Histological image of the rectangular tissue region in (a). The upper part is the necrotic tissue without nuclei, and the lower part is the viable cancerous tissue. (c) The average reflectance spectra of the tumor, necrosis, and normal tissue with std. The red solid line represents the average spectra of cancerous tissue, and the blue dotted line represents the average spectra of the normal tissue. The green dashed line represents the average spectra of the necrotic tissue. The error bars are the std at a certain wavelength of the three curves.

Figure 7 shows an RGB composite color image of an example hypercube. The tumor exhibited a white necrotic appearance, which was confirmed by the histological image in the interface between viable and necrotic tumor tissue. The reflectance spectrum of a tumor with necrosis differs from that of a tumor without necrosis. The image also shows that the pre-existing blood vessels in normal tissue reached the tumor region and grew new blood vessels into cancerous tissue. The highly vascularized tumor region grew well due to adequate oxygen and nutrient supply and due to removal of waste through blood vessels, while the nonvascularized tumor region became necrotic due to the lack of vessels. These observations were 
consistent with their spectra curves (Fig. 7). The viable cancer tissue is heterogeneous with higher spectra variations compared to the necrotic tissue. The average reflectance spectra of the viable tumor were lower than those of the normal tissue, which reflected the higher amount of hemoglobin in the highly vascularized cancer tissue.

Figure 8 shows the visualization of all the spectral features explored in this study, which include the RGB composite image of hypercube, and the mean, std, and sum of the selected tumor and normal tissue regions of interest (ROI). The texture of the tissue could be clearly visualized from the std image. The mean and sum image only differ by a constant. Due to the large dynamic range of the spectra along the wavelength, the average of the hypercube in the ROI did not reflect the correlation with the RGB image. All these features captured different aspects of the differences between tumor and normal tissue, which are visualized in these figures.

\subsection{Feature Selection and Classification Results}

The objective of the feature selection is to identify the features which are critical to minimize the classification error. The mRMR feature selection method selected a compact feature set with the $\mathrm{mR}$ to the target class and the MR within the feature set. Figure 9 shows the mutual information of the extracted spectral features with the class labels (tumor or normal), which reflects the relevance of each feature with respect to the class labels. The highest mutual information was achieved by FCs. Normalized reflectance above $850 \mathrm{~nm}$ showed higher relevance than other wavelengths. Derivatives, mean, std, and
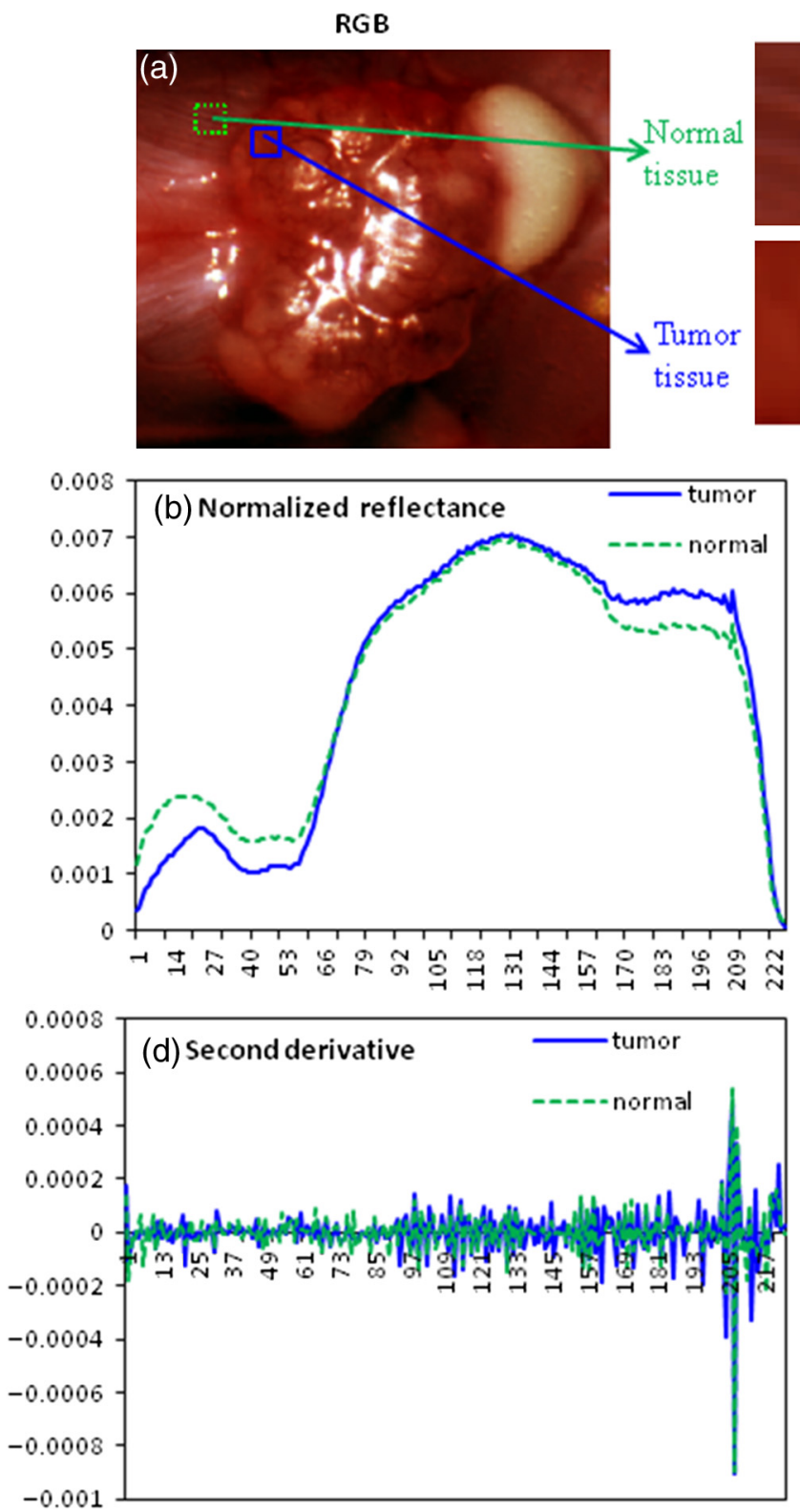

Tumor

tissue
RGB
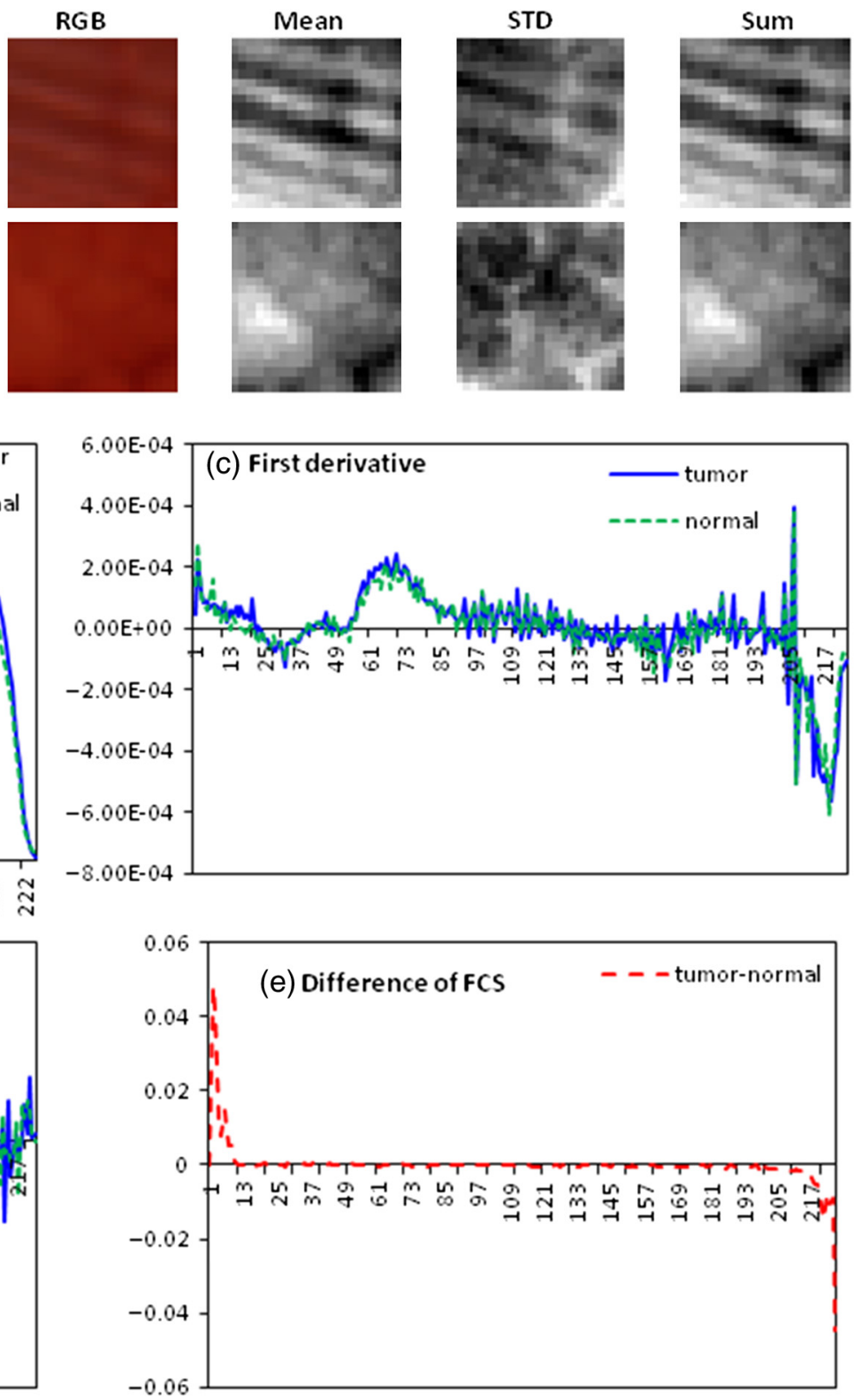

Fig. 8 Feature extraction and visualization. (a) RGB composite image of hypercube, mean, std, and sum of the selected tumor and normal tissue ROI. (b)-(e) Average normalized reflectance curve, first derivative, second derivative, and the difference of FCS between normal and tumor tissue in the selected $\mathrm{ROI}$ in (a). 


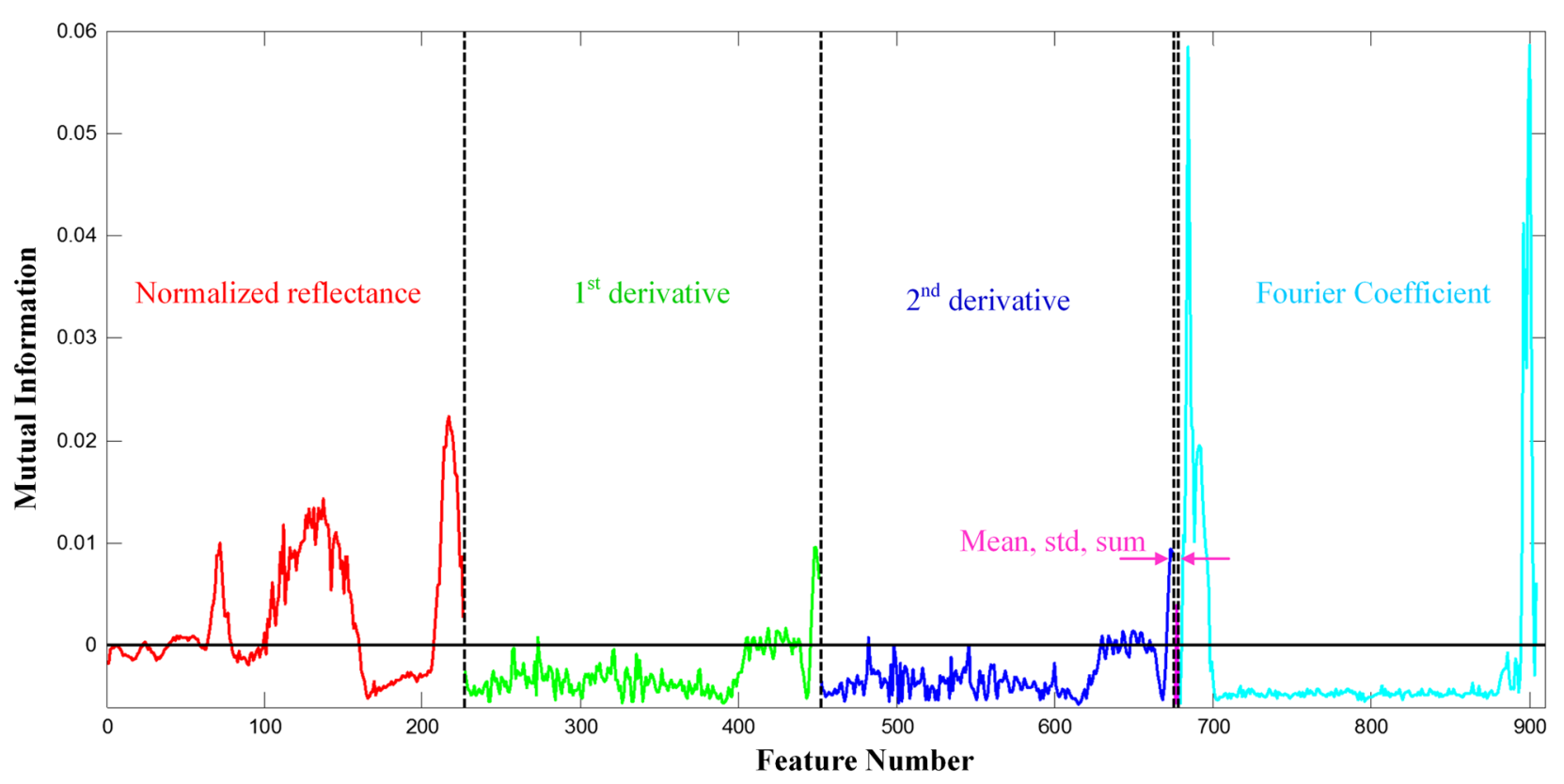

Fig. 9 Mutual information between features and class labels. The $x$ axis represents the feature number, and the $y$ axis represents the mutual information.

sum generally exhibited lower mutual information with class labels.

Figure 10 displays visualization of the mutual information among individual features, which represents the redundancy within the 904 features. It can be seen clearly that the lower left square of size $226 \times 226$ shows relatively higher mutual information than other regions, which demonstrates that wavelength features were highly correlated with each other. Therefore, the normalized intensity values across the wavelength range contain complementary information as well as redundancies.

Figure 11 shows the evaluation of feature sets of different sizes by supervised classification. The metrics initially increased with the feature number, reached a maximum, and then decreased as the feature set went to its maximum size of 904 .

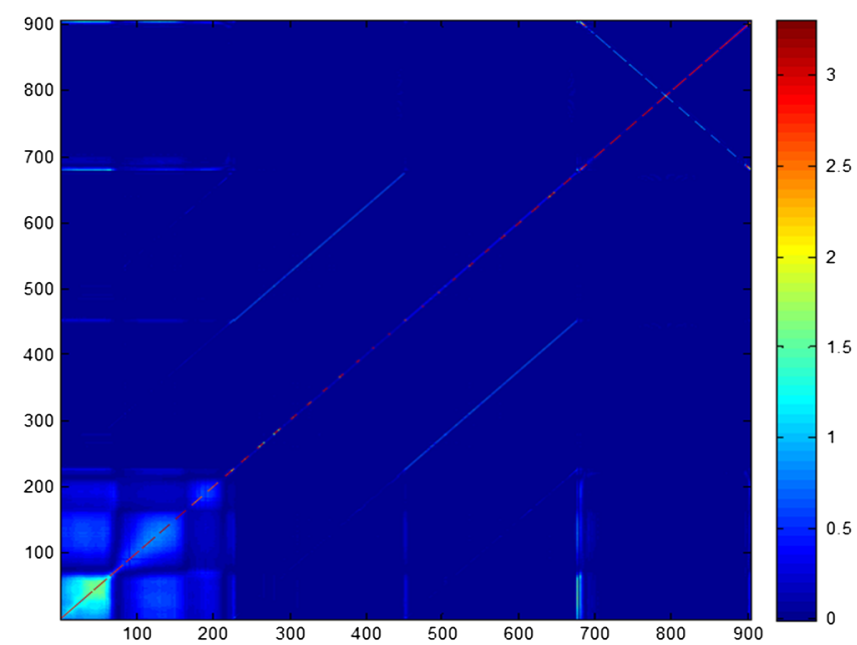

Fig. 10 Mutual information between individual features. Color bar on the right shows the color map corresponds to the value of mutual information. Higher mutual information indicates more redundancy between features.
Classification with the full feature set of dimension 904 was not as good as the feature set of dimension 20. We found that a feature set of size 20 gave the best performance, with an average accuracy, sensitivity, and specificity of $67.2 \%, 77.5 \%$, and $54.0 \%$, respectively. In this experiment, classification was used only for evaluating and comparing the effectiveness of feature sets of different sizes. Since the focus of this study was not on classification, we will try to improve classification in the future.

As shown in Fig. 11, we have run mRMR with cross validation to select the optimal feature set $\mathrm{F}=\left\{f_{i}\right\}_{i=1,2, \ldots m}$ of size $m(m=1,3,5, \ldots, 750,904)$ from 904 features. We have generated a series of feature rankings, $\left\{f_{1}\right\},\left\{f_{1}, f_{2}, f_{3}\right\}, \ldots$, $\left\{f_{1}, f_{2}, \ldots, f_{750}\right\},\left\{f_{1}, f_{2}, \ldots, f_{904}\right\}$, where $f_{i}$ represents the feature that has been ranked in the $i$ th position of each optimal

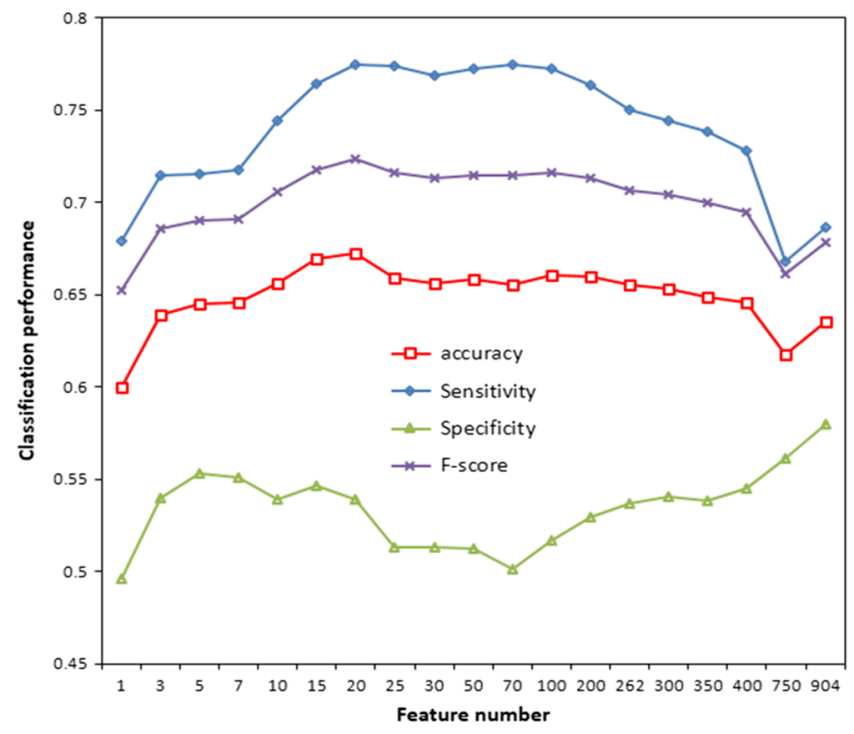

Fig. 11 Feature selection and classification. 


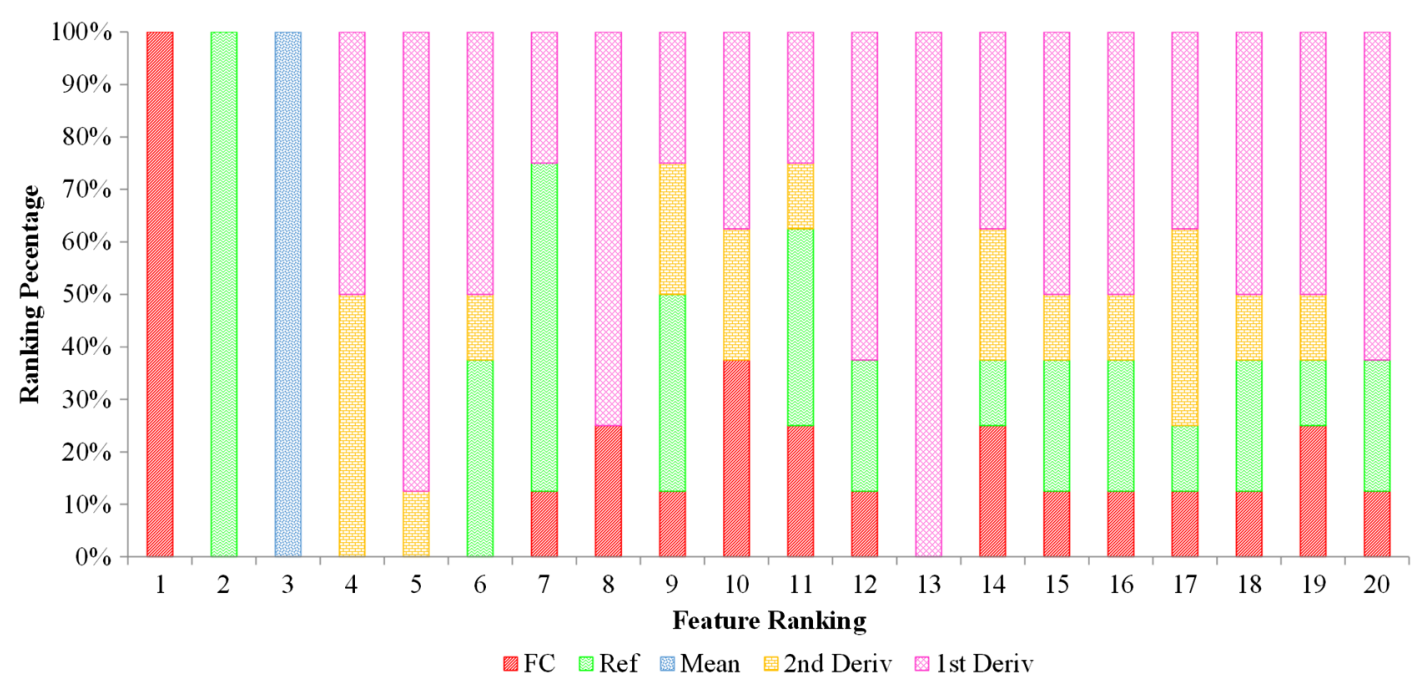

Fig. 12 Feature ranking. The $x$ axis is the ranking from 1 to 20 , and the $y$ axis is the percentage of the selection frequency for different features on each rank. Each bar represents the normalized frequency of different feature types being selected as the ith ranked feature.

feature set. It should be noted that $f_{i}$ could be different on different cross validation folds or different among optimal feature sets of different sizes. Therefore, we can analyze the composition of the $i$ th ranked feature $f_{i}$ and count the ranking frequency of individual features. Figure 12 summarizes the ranking frequency of different feature types. Each bar represents the normalized frequency of different feature types being selected as the $i$ th ranked feature $(i=1,2, \ldots, 20)$. FC is the only selected top ranking feature type, normalized reflectance is the only feature type that ranked the second, and the mean spectrum is the thirdranked feature type. Spectral derivatives ranked fourth and fifth even though their mutual information with corresponding labels was low. This could be explained by the fact that mRMR selects not only the features with high mutual information but also with low redundancy within the feature set.

\section{Discussion}

Complete excision of tumors remains one of the key challenges in tumor surgery. Failure to remove all tumor cells increases the risk of tumor recurrence and the need for secondary surgery. Current intraoperative margin assessment is performed by visual inspection and palpation, followed by examination of suspicious areas using histopathological evaluation. Intraoperative cancer imaging and diagnosis is valuable to surgeons in the evaluation and excision of sequential layers of tissue. We developed preprocessing techniques and explored the relevance of different spectral features on tissue labels and redundancy among individual features. Although the proposed method was tested in an artificial animal model, the preprocessing and feature analysis techniques should also work well for the real clinical situation, since the glares, motion artifacts, curvature, and high-dimensionality problems may exist in any intraoperative hyperspectral images. We showed that the combination of FC, normalized reflectance, mean, and spectral derivatives could improve the distinction between cancerous and normal tissue. Our longterm goal is to provide visual guidance during surgery to aid tumor margin evaluation, thereby decreasing the amount of tumor left behind and increasing tumor-free survival. Although we used an open operative technique, this technology should be well suited to laparoscopic and robotic surgery by coupling the HSI instrument with a laparoscope.

The key advantage of HSI for intraoperative tumor visualization is that it acquires images of a large area of tissue in a noncontact manner and generates objective tumor maps to enable the surgeon to excise and evaluate sequential layers of tissue during surgery without the need for tissue sectioning and staining. The penetration depth of the HSI under $900 \mathrm{~nm}$ does not exceed a few millimeters, which is adequate for the sequential imaging and diagnosis during surgery. This technique could provide fast and objective feedback to the surgeon as to whether all tumor tissue has been excised or whether further tissue removal is required. Although this study used head and neck cancer as an example, HSI may be used to provide intraoperative diagnosis and ensure a clear margin during tissue-conserving surgery for other cancer types.

One of the common problems during intraoperative imaging is that glare caused by specular reflection of the liquid on the tissue surface is always present in the surgical images, which deteriorates the quality of optical imaging and affects the quantitative analysis of the images. Cross-polarization has been widely used for glare removal during the imaging stage by placing a linear polarizer in front of the lighting and another orthogonal polarizer in front of the detector. The orthogonal polarization has the effect of selectively detecting photons that have undergone multiple scattering events in the tissue and rejecting the photons from specular reflection from tissue surface. As there are no cross polarizers in our imaging instrument, we developed a simple but effective method to remove glare pixels, and thus the diagnostic information contained in these pixels is also lost during the process. To the best of our knowledge, this is the first time that a fast and efficient glare detection method has been designed specifically for intraoperative hyperspectral images. The proposed glare removal method can also be applied to other optical images for eliminating glares.

In this study, GFP fluorescence images of tumors are used as the in vivo gold standard for tumor margin assessment. It was demonstrated that under white light illumination, spectral images at the GFP emission peaks do not show higher contrast 
than images at other wavelengths; therefore, the GFP signal does not contribute to the differentiation of tumor and normal tissue by spectral-spatial classification. In the future, ex vivo histopathological images can be further registered to the in vivo images in order to provide a more detailed diagnostic map.

One of the challenges in applying medical HSI for surgical guidance is that the imaging and diagnosis procedures should be done in a clinically useful time frame. ${ }^{34}$ The imaging instrument used in this study usually takes about $2 \mathrm{~min}$ to acquire $226 \mathrm{spec}-$ tral images from 450 to $900 \mathrm{~nm}$ with a $2-\mathrm{nm}$ increment, and the image testing takes about $1 \mathrm{~min}$. The time cost for evaluating tissues during surgery is significantly reduced compared to the traditional histopathology (20 min to an hour). The diagnosis with HSI was performed by quantitative image analysis. High feature dimensionality would lead to more computation cost; therefore, it is essential to extract only the most relevant features for diagnosis. It should be noted that the $m$ best features are not the best $m$ features, which means that the combinations of individually good features do not necessarily lead to good classification performance. Therefore, the best 20 features were not the 20 best features with highest mutual information. The best feature set should be relevant to the class labels as well as complementary to each other. Although only spectral features were used in this study, spatial features such as texture descriptors could be incorporated to further improve the classification performance. In addition to mutual information, similarity metrics such as the F-score and Pearson's correlation coefficient can also be used to characterize the relevance and redundancy. With the advancement of the HSI hardware and software, the imaging speed and image analysis time may be further reduced. Therefore, HSI is promising for intraoperative tumor margin delineation and visualization. In the future, we will proceed to evaluate the ability of HSI for residual tumor detection, which is also a key issue affecting patient prognosis.

\section{Conclusions}

We developed a set of preprocessing techniques for improving the quality of intraoperative HSI data. We also evaluated our feature extraction and a set of selection methods for differentiating cancer from normal tissue using this head and neck cancer model. We further demonstrated the potential of HSI as a noninvasive tool for tumor visualization and classification during surgery in an animal study. We believe that the HSI and quantitative analysis methods could have wide applications in future medical practice when more fully implemented into clinical devices.

\section{Acknowledgments}

This research is supported in part by NIH grants (R01CA156775 and R21CA176684), Georgia Research Alliance Distinguished Scientists Award, Emory SPORE in Head and Neck Cancer (NIH P50CA128613), and Emory Molecular and Translational Imaging Center (NIH P50CA128301).

\section{References}

1. R. L. Siegel, K. D. Miller, and A. Jemal, "Cancer statistics, 2015," CA Cancer J. Clin. 65(1), 5-29 (2015).

2. T. J. Smith and B. E. Hillner, "Bending the cost curve in cancer care," N. Engl. J. Med. 364(21), 2060-2065 (2011).

3. Q. T. Nguyen et al., "Surgery with molecular fluorescence imaging using activatable cell-penetrating peptides decreases residual cancer and improves survival," Proc. Natl. Acad. Sci. U. S. A. 107(9), 4317-4322 (2010).

4. S. Keereweer et al., "Image-guided surgery in head and neck cancer: current practice and future directions of optical imaging," Head and Neck 34(1), 120-126 (2012).

5. R. H. Spiro et al., "Pattern of invasion and margin assessment in patients with oral tongue cancer," Head and Neck 21(5), 408-413 (1999).

6. L. Jacobs, "Positive margins: the challenge continues for breast surgeons," Ann. Surg. Oncol. 15(5), 1271-1272 (2008).

7. R. Haque et al., "Surgical margins and survival after head and neck cancer surgery," BMC Ear Nose Throat Disord. 6(2), 1-6 (2006).

8. F. Meric et al., "Positive surgical margins and ipsilateral breast tumor recurrence predict disease-specific survival after breast-conserving therapy," Cancer 97(4), 926-933 (2003).

9. S. Keereweer et al., "Optical image-guided surgery-where do we stand?," Mol. Imaging Biol. 13(2), 199-207 (2011).

10. R. F. Gandour-Edwards, P. J. Donald, and D. A. Wiese, "Accuracy of intraoperative frozen section diagnosis in head and neck surgery: experience at a university medical center," Head and Neck 15(1), 33-38 (1993).

11. H. Akbari et al., "Detection of cancer metastasis using a novel macroscopic hyperspectral method," Proc. SPIE 8317, 831711 (2012).

12. H. Akbari et al., "Hyperspectral imaging and quantitative analysis for prostate cancer detection," J. Biomed. Opt. 17(7), 076005 (2012).

13. R. Pike et al., "A minimum spanning forest based hyperspectral image classification method for cancerous tissue detection," Proc. SPIE 9034, 90341W (2014).

14. G. Lu et al., "Hyperspectral imaging for cancer surgical margin delineation: registration of hyperspectral and histological images," Proc. SPIE 9036, 90360S (2014).

15. G. Lu et al., "Quantitative wavelength analysis and image classification for intraoperative cancer diagnosis with hyperspectral imaging," Proc. SPIE 9415, 94151B (2015).

16. G. Lu et al., "Estimation of tissue optical parameters with hyperspectral imaging and spectral unmixing," Proc. SPIE 9417, 94170Q (2015).

17. S. V. Panasyuk et al., "Medical hyperspectral imaging to facilitate residual tumor identification during surgery," Cancer Biol. Ther. 6(3), 439-446 (2007).

18. S. Kiyotoki et al., "New method for detection of gastric cancer by hyperspectral imaging: a pilot study," J. Biomed. Opt. 18(2), 026010 (2013).

19. S. C. Gebhart, R. C. Thompson, and A. Mahadevan-Jansen, "Liquidcrystal tunable filter spectral imaging for brain tumor demarcation," Appl. Opt. 46(10), 1896-1910 (2007).

20. K. J. Zuzak et al., "Intraoperative bile duct visualization using nearinfrared hyperspectral video imaging," Am. J. Surg. 195(4), 491-497 (2008).

21. G. Lu and B. Fei, "Medical hyperspectral imaging: a review," J. Biomed. Opt. 19(1), 010901 (2014).

22. D. Wang et al., "The pivotal role of integrin $\beta 1$ in metastasis of head and neck squamous cell carcinoma," Clin. Cancer Res. 18(17), 4589-4599 (2012).

23. G. Lu et al., "Hyperspectral imaging for cancer surgical margin delineation: registration of hyperspectral and histological images," Proc. SPIE 9036 90360S (2014).

24. H. Lange, "Automatic glare removal in reflectance imagery of the uterine cervix," Proc. SPIE 5747, 2183-2192 (2005).

25. E. Claridge and D. Hidovic-Rowe, "Model based inversion for deriving maps of histological parameters characteristic of cancer from ex-vivo multispectral images of the colon," IEEE Trans. Med. Imaging $\mathbf{3 3}(4)$, 822-835 (2014).

26. K.-M. Saipullah and D.-H. Kim, "Target detection of hyperspectral images based on their Fourier spectral features," Opt. Eng. 51(11), 111704 (2012).

27. R. Pourreza-Shahri et al., "Classification of ex-vivo breast cancer positive margins measured by hyperspectral imaging," in 20th IEEE Int. Conf. on Image Processing (ICIP), pp. 1408-1412 (2013).

28. G. Hughes, "On the mean accuracy of statistical pattern recognizers," IEEE Trans. Inf. Theory 14(1), 55-63 (1968).

29. H. Peng, L. Fulmi, and C. Ding, "Feature selection based on mutual information criteria of max-dependency, max-relevance, and min-redundancy," IEEE Trans. Pattern Anal. Mach. Intell. 27(8), 1226-1238 (2005). 
30. G. Lu et al., "Spectral-spatial classification for noninvasive cancer detection using hyperspectral imaging," J. Biomed. Opt. 19(10), 106004 (2014).

31. G. Lu et al., "Spectral-spatial classification using tensor modeling for cancer detection with hyperspectral imaging," Proc. SPIE 9034, 903413 (2014).

32. N. Otsu, "A threshold selection method from gray-level histograms," IEEE Trans. Syst. Man Cybern. 9(1), 62-66 (1979).

33. J. N. Kapur, P. K. Sahoo, and A. K. C. Wong, "A new method for graylevel picture thresholding using the entropy of the histogram," Comput. Vision Graph Image Process. 29(3), 273-285 (1985).

34. N. Lue et al., "Portable optical fiber probe-based spectroscopic scanner for rapid cancer diagnosis: a new tool for intraoperative margin assessment," PLoS One 7(1), e30887 (2012).
Guolan Lu is a PhD candidate in the Department of Biomedical Engineering at the Georgia Institute of Technology and Emory University, Atlanta, Georgia.

Baowei Fei is an associate professor in the Department of Radiology and Imaging Sciences at Emory University and in the Department of Biomedical Engineering at Emory University and the Georgia Institute of Technology. He received his MS and PhD degrees from Case Western Reserve University, Cleveland, Ohio. He is a Georgia Cancer Coalition distinguished scholar and director of the Quantitative Bioimaging Laboratory (www.feilab.org) at Emory University School of Medicine.

Biographies for the other authors are not available. 